\title{
A prospective study of ventilated neonates in a tertiary care hospital in Sri Lanka compared to retrospective data from the same unit
}

\author{
M N Lucas ${ }^{1}$, M Weerasekera ${ }^{2}$ \\ Sri Lanka Journal of Child Health, 2013; 42(1): 10-19
}

\begin{abstract}
Objectives: To prospectively study several aspects of ventilated neonates at the neonatal intensive care unit (NICU) of Sri Jayawardenepura General Hospital (SJGH) and compare this data to retrospective data from the same unit.
\end{abstract}

Method: A descriptive observational, longitudinal hospital based prospective study was conducted on ventilated babies in NICU, SJGH from1st July 2009 to 1st July 2010. Data were obtained using a pretested recording form. NICU records were used to gather data of infants ventilated from $1^{\text {st }}$ July 2000 to $1^{\text {st }}$ July 2001. Data obtained from the current study were compared to data in 2000/2001. Data were analysed using SPSS version 16 for Windows.

Results: During the study period 135 babies were ventilated. Four were excluded due to severe congenital defects. Seventy two percent were male and $53 \%$ had gestational periods of 32 weeks or less. There were $46 \%$ very low birth weight (VLBW) babies. In $72 \%$ the indication for ventilation was respiratory distress syndrome (RDS). Duration of ventilation was over one week in 34\%. Continuous positive airway pressure (CPAP) was the sole mode of ventilation in $33 \%$. Surfactant was used in $53 \%$ babies, $96 \%$ for RDS. Oxygen for over 2 weeks was required in $17 \%$ and $22 \%$ received theophylline as respiratory stimulants. Midazolam infusion was used for sedation in $56 \%$. Total parenteral nutrition was started in $56 \%, 29 \%$ received blood transfusions and $65 \%$ received volume support or inotropes for hypotension. Complications included seizures (16\%), persistent pulmonary hypertension of the newborn $(9 \%)$, patent ductus arteriosus $(8 \%)$, pulmonary haemorrhage $(7 \%)$, retinopathy of prematurity $(6 \%)$,

${ }^{1}$ Lecturer, Department of Paediatrics, Faculty of Medicine, University of Colombo

${ }^{2}$ Consultant Neonatologist, Sri Jayawardenepura General Hospital, Kotte

(Received on 20 July 2012: Accepted after revision on 24 August 2012) nosocomial sepsis $(6 \%)$, ventilator-associated pneumonia (5\%), bronchopulmonary dysplasia (5\%), necrotising enterocolitis (3\%), intraventricular haemorrhage $(2 \%)$ and pneumothorax (2\%). Eighty eight percent babies were followed up till 2 years of age. Mortality in $2009 / 2010$ was $18 \%$ compared to $39 \%$ in $2000 / 2001(\mathrm{P}<0.0001)$.

Conclusions: Babies with 32 weeks or less gestation and VLBW babies were significantly more in 2009/2010 $(\mathrm{P}<0.05)$. Complications such as VAP, nosocomial sepsis and pneumothorax were significantly more common in babies ventilated in $2000 / 2001$ but ROP was significantly more common in babies ventilated in 2009/2010 $(\mathrm{P}<0.05)$. HIE caused significantly more deaths in 2000/ 2001 whilst significantly more deaths occurred in ex-utero babies and babies with pulmonary haemorrhage in $2009 / 2010(P<0.05)$. Overall mortality, mortality in babies with 32 weeks or less gestation and mortality in VLBW babies were significantly lower in $2009 / 2010(\mathrm{P}<0.0001)$. In 2009/2010, the outcome in babies receiving CPAP only was significantly better than those receiving IMV only $(\mathrm{P}<0.0001)$. There was no morbidity at 2 years of age in significantly more babies over 32 weeks gestation in 2009/2010 compared to babies with gestation 32 weeks or less $(\mathrm{P}<0.0001)$.

(Key words: Ventilated neonate; prospective study; Sri Lanka)

\section{Introduction}

NICU of SJGH admits sick and premature neonates from SJGH and accepts ex-utero transfers. It is the training centre in neonatal intensive care. It has 7 ventilators (3 SLE 2000, 2 Infant star 200, 1 Bear cub 750 VS and 1 Bear cub 750 PSV), all having continuous positive airway pressure (CPAP) and intermittent mandatory ventilation (IMV) modes, some also with synchronized intermittent mandatory ventilation (SIMV). Neither volume cycled ventilation nor high frequency ventilation are available. 
An extensive online literary search using the key words 'ventilated neonate', did not reveal any prospective studies on ventilated neonates anywhere in the world. However, there were retrospective studies ${ }^{1,2}$. In Sri Lanka, 2 such studies were presented at the $12^{\text {th }}$ Asia Pacific Congress of Paediatrics ${ }^{3}$ and the $6^{\text {th }}$ Annual Scientific Sessions of the Perinatal Society of Sri Lanka ${ }^{4}$. There are no other Sri Lankan studies on ventilated neonates and the present study was designed to address this deficiency.

\section{Objectives}

1. To prospectively study several aspects of ventilated neonates at the NICU, SJGH, such as gender difference, indications for ventilation, type and duration of ventilation, surfactant use, duration of oxygen therapy, use of muscle relaxants and sedation, complications of CPAP and IMV, incidences of ventilator associated pneumonia and colonization of the respiratory tract, other ventilation-related complications, weaning strategy, use of respiratory stimulants, need for re-intubation, use of parenteral nutrition, need for blood transfusion, incidence and treatment of hypotension, mortality, causes of death and short-term morbidity

2. To compare data from the current study with retrospective data from the same unit from $1 \mathrm{st}$ July 2000 to 1 st July 2001.

\section{Method}

A descriptive observational, longitudinal, hospital based prospective study was conducted in NICU, SJGH from 1st July 2009 to 1st July 2010 on all ventilated babies, including ex-utero babies, but excluding babies with severe congenital anomalies. Data were obtained by the first author using a pretested recording form.

Several infection control policies are practised at NICU to minimise nosocomial infections. Hand washing was strictly enforced, a 2 minute hand washing before entering, followed by 20 second hand washing in between patients. Hand washing was done even if an inanimate object was touched before seeing a patient and also before any procedure. No hand accessories were worn by the staff. Clothes were folded above the elbow and a sterile gown and shoes worn prior to entry. Only mothers of admitted babies were allowed inside the NICU. Bacterial filters were used for both inspiratory and expiratory limbs, sterile water was used for the humidification systems, condensed water in the circuits was drained and circuits changed weekly. Disposable sterile suction catheters were used. Sterile needles were used for punctures. Orotracheal rather than nasotracheal tubes were used for intubation. Probes were disinfected. One to one nursing with barrier precautions was used whenever possible.

Period of gestation (POG) was estimated according to mother's obstetric record when supported by an obstetric ultrasound at 15-19 weeks. Otherwise, maturity assessment by the first author according to the new Ballard score was taken as the POG.

Respiratory distress syndrome (RDS) was diagnosed when the following were present within 4 hours of birth: respiratory rate over 60/minute, intercostal/ subcostal/sternal retractions, expiratory grunt and chest $\mathrm{x}$-ray (CXR) having a ground glass appearance with air bronchogram / hazy appearance or white out appearance ${ }^{5}$. Hypoxic ischaemic encephalopathy (HIE) was diagnosed if the following were present: acidaemia, Apgar score 0-3 for over 5 minutes, neurological manifestations and multisystemic organ dysfunction ${ }^{6}$. Persistent pulmonary hypertension of the newborn (PPHN) was diagnosed when an infant with an echocardiographically confirmed structurally normal heart had the following: severe hypoxaemia $\left(\mathrm{PaO}_{2}<45 \mathrm{mmHg}\right)$, hypoxaemia disproportionate to clinical and CXR findings, acid base abnormalities and a right to left shunt via ductus or foramen ovale ${ }^{5}$. Meconium aspiration syndrome (MAS) was defined as respiratory distress in an infant born through meconium stained amniotic fluid whose symptoms could not be otherwise explained ${ }^{7}$. Sepsis was diagnosed if blood cultures were positive in a baby with suggestive clinical features.

Ventilator associated pneumonia (VAP) was defined as follows: patients mechanically ventilated for 48 or more hours having an abnormal CXR with at least one of the following: new or progressive and persistent infiltrate, consolidation, cavitation and/or pneumatoceles in addition to: worsening gas exchange and at least 3 of the following: temperature instability with no other recognized cause, increased respiratory secretions, increased suctioning requirements, apnoea, tachypnoea, nasal flaring with chest wall retraction or grunting, wheezing, cough and bradycardia $(<100$ beats/min) or tachycardia $(>170 \text { beats } / \mathrm{min})^{8}$.

Colonisation of trachea without sepsis implied organisms isolated in endotracheal tube (ETT) secretions without systemic evidence of sepsis and negative blood cultures. Colonisation of trachea with sepsis implied organisms isolated on ETT secretions 
with systemic evidence of sepsis with or without positive blood cultures. Patent ductus arteriosus (PDA) was diagnosed clinically supported by echocardiography. Retinopathy of prematurity (ROP) was diagnosed by one of the 2 consultant eye surgeons at SJGH when babies 34 weeks or less in gestation or birth weight less than $1500 \mathrm{~g}$ were screened. Intraventricular haemorrhage (IVH) was diagnosed according to brain ultrasound scan performed by neonatologist or consultant radiologist. Bronchopulmonary dysplasia (BPD) was defined as babies needing over $21 \%$ oxygen at 28 days of age 9 .

NICU records were used to gather data regarding infants ventilated from $1^{\text {st }}$ July 2000 to $1^{\text {st }}$ July 2001 . All babies were seen by the first author during the study period. They were followed up in the clinic for 2 years.

Data obtained from the current study were compared to data of babies ventilated in $2000 / 2001$.

Data were analysed using SPSS version 16 for Windows. Pearson's Chi Square Test was the statistical test used.

Ethical clearance for the study was obtained from the Ethics Review Committee of the Sri Lanka College of Paediatricians. Permission was obtained from the Director, SJGH prior to commencement of study. Informed consent was obtained from parents for the study.

\section{Results}

One hundred and thirty five babies were ventilated. Two babies with antenatally diagnosed hydrocephalus, a baby with complex cyanotic heart disease and a baby with meningocele and hydrocephalus were excluded. Ninety four (72\%) were male. Distributions of ventilated babies in 2009/2010 and 2000/2001 according to their POG are shown in Table 1.

Table 1: Distribution of ventilated babies

\begin{tabular}{|ccc|}
\hline POG & $\mathbf{2 0 0 9 / 2 0 1 0}$ & $\mathbf{2 0 0 0} / \mathbf{2 0 0 1}$ \\
(weeks) & $n=131$ & $n=122$ \\
& No. (\%) & No. (\%) \\
Less than 28 & $19(14.5)$ & $25(20.5)$ \\
$28-32$ & $51(39)$ & $25(20.5)$ \\
$33-36$ & $25(19)$ & $22(18)$ \\
More than 36 & $36(27.5)$ & $50(41)$ \\
\hline
\end{tabular}

There were significantly more babies with POG of 32 weeks or less in 2009/2010 compared to 2000/2001 $(\mathrm{P}=0.047)$. Thirty eight $(29 \%)$ babies were small for gestational age in 2009/2010 compared to $33(27 \%)$ in $2000 / 2001(\mathrm{P}=0.729)$. Twenty $(15 \%)$ babies had birth weights $<1000 \mathrm{~g}$ in $2009 / 2010$ compared to 15 $(12 \%)$ in $2000 / 2001 \quad(\mathrm{P}=0.494)$. There were significantly more $(60,46 \%)$ very low birth weight (VLBW) babies $(<1500 \mathrm{~g})$ in $2009 / 2010$ compared to (40,33\%) VLBW babies in $2000 / 2001(\mathrm{P}=0.034)$. Indications for ventilation in 2009/2010 and 2000/2001 are shown in Table 2.

Table 2: Indications for ventilation in 2009/2010 and 2000/2001

\begin{tabular}{|c|c|c|c|}
\hline & $\begin{array}{c}\mathbf{2 0 0 9} / \mathbf{2 0 1 0} \\
n=131 \\
\text { No. }(\%)\end{array}$ & $\begin{array}{c}2000 / 2001 \\
n=122 \\
\text { No. }(\%)\end{array}$ & P value \\
\hline Respiratory distress syndrome & $95(72)$ & $79(65)$ & 0.183 \\
\hline Hypoxic ischaemic encephalopathy & $08(06)$ & $09(07)$ & 0.687 \\
\hline Meconium aspiration syndrome & $04(03)$ & $12(10)$ & 0.027 \\
\hline Congenital pneumonia & $14(11)$ & $08(07)$ & 0.244 \\
\hline Persistent pulmonary hypertension of the newborn & $05(04)$ & $01(01)$ & 0.117 \\
\hline Sepsis & $01(01)$ & 0 & 0.334 \\
\hline Apnoea & $02(02)$ & $04(03)$ & 0.360 \\
\hline Transient tachypnoea of the newborn & $02(02)$ & $01(01)$ & 0.604 \\
\hline Pulmonary haemorrhage & 0 & $01(01)$ & 0.299 \\
\hline Myopathy & 0 & $01(01)$ & 0.299 \\
\hline Postmeningocele surgery & 0 & $01(01)$ & 0.299 \\
\hline Congenital infection & 0 & $01(01)$ & 0.299 \\
\hline Unknown & 0 & $02(02)$ & 0.141 \\
\hline Congenital diaphragmatic hernia & 0 & $01(01)$ & 0.299 \\
\hline Intracranial haemorrhage & 0 & $01(01)$ & 0.299 \\
\hline
\end{tabular}


MAS was seen in significantly more babies in $2000 / 2001$ than in $2009 / 2010(P=0.027)$. Group B streptococcus was isolated in all 14 babies with congenital pneumonia in the 2009/2010 group and 6 of 8 babies in the $2000 / 2001$ group.

Durations of ventilation in 2009/10 and 2000/2001 are shown in Table 3.

Table 3: Duration of ventilation in 2009/2010 and 2000/2001

\begin{tabular}{|cccc|}
\hline $\begin{array}{c}\text { Duration of } \\
\text { ventilation }\end{array}$ & $\begin{array}{c}\mathbf{2 0 0 9 / 2 0 1 0} \\
n=131 \\
\text { No. }(\%)\end{array}$ & $\begin{array}{c}\mathbf{2 0 0 0 / 2 0 0 1} \\
n=122\end{array}$ & P value \\
No. $(\%)$ & \\
$<24$ hours & $12(09)$ & $06(05)$ & 0.190 \\
$24-48$ hours & $14(11)$ & $10(08)$ & 0.499 \\
3-7 days & $60(46)$ & $53(43)$ & 0.706 \\
$>7$ days & $45(34)$ & $53(43)$ & 0.138 \\
\hline
\end{tabular}

There were no statistically significant differences between the 2 groups with regard to duration of ventilation.

Surfactant was unavailable for use in 2000/2001. In $2009 / 2010$ surfactant was used in 69 babies, RDS being the commonest indication (66/69), other indications being MAS (1/4), congenital pneumonia $(1 / 14)$ and pulmonary haemorrhage $(1 / 2)$. Of the 94 babies ventilated for RDS, 65 received surfactant. Significantly more babies $(54,77 \%)$ in the 32 week or less age group received surfactant compared to babies $(17,26 \%)$ in the over 32 week age group $(\mathrm{P}=0.002)$.

Surfactant was used only as rescue therapy for babies with RDS. Fifty three were given surfactant for persistent or worsening respiratory distress or oxygen requirement exceeding 30\% while on CPAP. Eight babies, 32 weeks of gestation or less, who required intubation at birth, were given surfactant as soon as it was available. Eight babies were given surfactant after developing persistent or worsening respiratory distress while on head box oxygen. Mean age of surfactant administration was $9 \pm 9.9$ hours. Of 66 babies admitted on day one of age, 65 received surfactant within the first 24 hours. The 2 babies receiving surfactant after 24 hours of age were both ex-utero transfers.

All babies were ventilated invasively via ETT in $2000 / 2001$. Types of ventilation used in the 2009/10 along with the outcomes are shown in Table 4.

Table 4: Types of ventilation used in 2009/2010 with outcome

\begin{tabular}{|lccc|}
\hline \multicolumn{1}{|c}{ Type of ventilation } & No. (\%) & Died (\%) & Survived (\%) \\
& $n=131$ & $n=24$ & $n=107$ \\
Continuous positive airway pressure (CPAP) only & $43(33)$ & $0(0)$ & $43(100)$ \\
Intermittent mandatory ventilation (IMV) only & $18(14)$ & $15(83)$ & $03(17)$ \\
CPAP followed by IMV & $50(38)$ & $09(18)$ & $41(82)$ \\
IMV followed by CPAP & $20(15)$ & $02(10)$ & $18(90)$ \\
\hline
\end{tabular}

Outcome in babies receiving CPAP only was significantly better than those receiving IMV only $(\mathrm{P}<0.0001)$, CPAP followed by IMV $(\mathrm{P}=0.003)$ or IMV followed by CPAP $(\mathrm{P}=0.035)$. There was no statistically significant difference whether CPAP was followed by IMV or IMV was followed by CPAP $(\mathrm{P}=0.406)$.

Sedation was given to babies seen to breathe against the ventilator and to those who were uncomfortable. Whilst 57 babies did not receive sedation, 54 were given midazolam infusion, 19 also receiving morphine and one also receiving phenobarbitone Muscle relaxants were not used. Only 2 complications were noted during CPAP application. One baby developed nasal irritation and 12 developed feed intolerance.
In 2009/2010, 62 babies required oxygen for less than a week and 8 for over 4 weeks but no baby required oxygen for over 8 weeks. Babies with POG 32 weeks or less required significantly longer periods of oxygen therapy compared to babies with POG over 32 weeks $(\mathrm{P}=0.048)$. Two ventilated babies developed pneumothorax. Maximum peak inspiratory pressure (PIP) used in them was $20-22 \mathrm{mmHg}$. There was no association between the occurrence of pneumothorax and the PIP used, in this study population.

Of 88 babies needing invasive ventilation, 24 (27\%) needed re-intubation. Re-intubation was required in 6/44 babies ventilated for 0-7 days compared to $18 / 44$ babies ventilated for over one week $(\mathrm{P}=0.004)$. In the 0-7 day group, 5 babies required one reintubation and one required 2. In the over one week group, 11 babies needed one re-intubation and 13 
needed 2-6. Significantly more babies in the over one week age group required 2 or more re-intubations compared to the 0-7 day group $(\mathrm{P}=0.026)$.

Fifty one babies receiving invasive ventilation were weaned off to nasal CPAP after extubation. Reduction of the pressure was the method used for CPAP weaning. Thirteen babies were extubated and connected to head box oxygen without connecting to CPAP. No baby received endotracheal CPAP. Weaning off to CPAP was the strategy used in a significantly higher number of babies 32 weeks or less POG compared to babies with POG over 32 weeks $(\mathrm{P}=0.019)$.

Respiratory stimulants, either oral theophylline or intravenous aminophylline, were given to 29 (22\%) babies, $97 \%$ of whom had a POG of 32 weeks or less.

Total parenteral nutrition (TPN) was commenced in $74(56 \%)$ babies. TPN was started in $55(74 \%)$ babies with a POG of 32 weeks or less compared to 19 $(26 \%)$ babies with a POG over 32 weeks $(\mathrm{P}<0.0001)$. TPN was used for a mean of $4 \pm 5$ days. No complications were encountered due to TPN.

The haemoglobin $(\mathrm{Hb})$ level was maintained above $14 \mathrm{~g} / \mathrm{dl}$ in all ventilated babies. Blood was transfused if the $\mathrm{Hb}$ level fell below $14 \mathrm{~g} / \mathrm{dl}$. Blood transfusions were given to $38(29 \%)$ ventilated babies, $71 \%$ of them having a POG of 32 weeks or less.

Blood pressure was maintained at more than the mean for gestational age by volume expansion and inotrope infusions. Eighty five $(65 \%)$ babies received either volume support or inotropes for hypotension. Of them, 54 had a POG of 32 weeks or less compared to 31 with a POG more than 32 weeks $(\mathrm{P}=0.006)$.

Complications noted prior to discharge in babies who were ventilated are shown in Table 5.

Table 5: Complications noted prior to discharge in ventilated babies

\begin{tabular}{|c|c|c|c|c|c|c|}
\hline Complication & $\begin{array}{c}\mathbf{2 0 0 9} / \mathbf{2 0 1 0} \\
n=131 \\
\text { No. }(\%)\end{array}$ & $\begin{array}{c}\mathbf{2 0 0 0 / 2 0 0 1} \\
n=122 \\
\text { No. }(\%)\end{array}$ & $\begin{array}{c}\text { Statistical } \\
\text { significance }\end{array}$ & $\begin{array}{c}\text { Survivals in } \\
2009 / 2010 \\
\text { No. }(\%)\end{array}$ & $\begin{array}{c}\text { Survivals in } \\
2000 / 2001 \\
\text { No. }(\%)\end{array}$ & $\begin{array}{c}\text { Statistical } \\
\text { significance }\end{array}$ \\
\hline VAP & $07(05)$ & $22(22)$ & $\mathrm{P}<0.001$ & $07(100)$ & $17(77)$ & $\mathrm{P}=0.000$ \\
\hline Nosocomial sepsis & $08(06)$ & $19(16)$ & $\mathrm{P}<0.05$ & $08(100)$ & $13(68)$ & $\mathrm{P}=0.197$ \\
\hline PDA & $11(08)$ & $11(09)$ & $\mathrm{P}=0.861$ & $11(100)$ & $11(100)$ & $\mathrm{P}=0.861$ \\
\hline Seizures & $21(16)$ & $13(11)$ & $\mathrm{P}=0.210$ & $14(66)$ & $06(46)$ & $\mathrm{P}=0.274$ \\
\hline IVH & $02(02)$ & $04(03)$ & $\mathrm{P}=0.120$ & $01(50)$ & $01(25)$ & $\mathrm{P}=0.419$ \\
\hline NEC & $04(03)$ & $04(03)$ & $\mathrm{P}=0.919$ & $04(100)$ & $01(25)$ & $\mathrm{P}=0.326$ \\
\hline PPHN & $12(09)$ & $05(04)$ & $\mathrm{P}=0.108$ & $08(66)$ & $02(40)$ & $\mathrm{P}=0.328$ \\
\hline Pulmonary haemorrhage & $09(07)$ & $02(02)$ & $\mathrm{P}<0.05$ & $03(33)$ & $01(50)$ & $\mathrm{P}=0.776$ \\
\hline Pneumothorax & $02(02)$ & $21(17)$ & $\mathrm{P}<0.001$ & 0 & $16(76)$ & $\mathrm{P}=0.000$ \\
\hline $\mathrm{BPD}$ & $07(05)$ & $03(03)$ & $\mathrm{P}=0.310$ & $06(86)$ & $01(33)$ & $\mathrm{P}=0.138$ \\
\hline ROP & $12(09)$ & $02(02)$ & $\mathrm{P}=0.009$ & $12(100)$ & $01(50)$ & $\mathrm{P}=0.011$ \\
\hline
\end{tabular}

$V A P=$ Ventilator associated pneumonia; $P D A=$ Patent ductus arteriosus; $\mathrm{IVH}=$ Intraventricular haemorrhage; $N E C=$ Necrotising enterocolitis; $P P H N=$ Persistent pulmonary hypertension of the newborn; $\mathrm{BPD}=$ Bronchopulmonary dysplasia; $R O P=$ Retinopathy of prematurity

VAP was seen in significantly more babies ventilated in $2000 / 2001$ compared to $2009 / 2010(\mathrm{P}<0.0001)$. In $2009 / 2010$, VAP was not seen in babies receiving CPAP. In babies ventilated invasively, incidence of VAP was $1 / 44$ in babies ventilated from 0-7 days compared to $6 / 44$ in babies ventilated for more than one week $(\mathrm{P}=0.049)$. In $2009 / 2010$ VAP was due to coliforms in 5 babies and acenetobacter in one baby.

The incidence of nosocomial sepsis was significantly more in babies ventilated in 2000/2001 $(\mathrm{P}<0.05)$. In 2009/2010, 8 (7.5\%) survivors developed nosocomial sepsis with positive blood cultures along with tracheal colonisation. Causative organisms were: coliforms (4), acenetobacter (2), staphylococcus (1) and candida (1). Sixteen (15\%) survivors developed tracheal colonisation without sepsis. ETT secretions were positive for acenetobacter (9), coliforms (5), pseudomonas (3) and candida (1). None of the 12 babies ventilated for less than 24 hours developed nosocomial sepsis.

In 2009/2010, one PDA required ligation. One baby with IVH had a grade 4 haemorrhage requiring 
evacuation due to midline shift. One baby with NEC required surgical intervention. One baby with BPD could not be weaned off and died on the ventilator. The other 6 babies had mild BPD and did not need oxygen on discharge.

Incidence of pneumothorax was significantly higher in $2000 / 2001$ compared to $2009 / 2010 \quad(\mathrm{P}<0.001)$. Incidence of ROP was significantly higher in 2009/2010 compared to 2000/2001 ( $P=0.009)$. Survival in babies with ROP was significantly higher in $2009 / 2010$ compared to $2000 / 2001(\mathrm{P}=0.011)$

The outcomes in 2009/2010 and 2000/2001 are shown in Table 6

Mortality in $2009 / 2010$ was significantly lower than in 2000/2001 $(\mathrm{P}<0.0001)$. There were $18(26 \%)$ deaths in 70 babies with a POG 32 weeks or less in
$2009 / 2010$ compared to $32(64 \%)$ deaths in 50 babies with a POG 32 weeks or less in 2000/2001 $(\mathrm{P}<0.0001)$. There were $19(32 \%)$ deaths in 60 VLBW babies in $2009 / 2010$ compared to $30(75 \%)$ deaths in 40 VLBW babies in 2000/2001 $(\mathrm{P}<0.0001)$.

Table 6: Outcome in 2009/2010 and 2000/2001

\begin{tabular}{|ccc|}
\hline Outcome & $\mathbf{2 0 0 9 / 2 0 1 0}$ & $\mathbf{2 0 0 0 / 2 0 0 1}$ \\
& $n=131$ & $n=122$ \\
& Number (\%) & Number (\%) \\
Deaths & $24(18)$ & $48(39)$ \\
Survivors & $107(82)$ & $74(61)$ \\
\hline
\end{tabular}

Ten babies were transferred ex-utero in 2009/2010 with $7(70 \%)$ deaths compared to 18 babies transferred ex-utero in 2000/2001 with $9(50 \%)$ deaths $(\mathrm{P}=0.047)$. Causes of death in $2009 / 2010$ and 2000/2001 are shown in Table 7.

Table 7: Causes of death in 2009/2010 and 2000/2001

\begin{tabular}{|lccc}
\hline \multicolumn{1}{|c}{ Causes of death } & $\mathbf{2 0 0 9 / 2 0 1 0}$ & $\mathbf{2 0 0 0 / 2 0 0 1}$ & $n=48$ \\
& $n=24$ & P value \\
Respiratory distress syndrome & $\mathbf{N o .}(\mathbf{\%})$ & No. (\%) & \\
Hypoxic ischaemic encephalopathy & $11(46)$ & $14(29)$ & 0.161 \\
Meconium aspiration syndrome & $01(04)$ & $09(19)$ & 0.092 \\
Congenital pneumonia & $0(0)$ & $03(06)$ & 0.211 \\
Pneumothorax & $0(0)$ & $01(02)$ & 0.476 \\
Other air leaks & $02(08)$ & $05(10)$ & 0.778 \\
Sepsis & $0(0)$ & $01(02)$ & 0.476 \\
Intraventricular haemorrhage & $0(0)$ & $06(13)$ & 0.070 \\
Persistent pulmonary hypertension of the newborn & $01(04)$ & $03(06)$ & 0.716 \\
Pulmonary haemorrhage & $04(17)$ & $03(06)$ & 0.160 \\
Necrotising enterocolitis & $06(25)$ & $01(02)$ & 0.002 \\
Bronchopulmonary dysplasia & $0(0)$ & $03(06)$ & 0.193 \\
Myopathy & $01(04)$ & $03(06)$ & 0.716 \\
Disseminated intravascular coagulation & $0(0)$ & $01(02)$ & 0.476 \\
Hydrops & $01(04)$ & $01(02)$ & 0.612 \\
Adrenal haemorrhage & $0(0)$ & $01(02)$ & 0.476 \\
Congenital diaphragmatic hernia & $0(0)$ & $02(04)$ & 0.310 \\
\end{tabular}

RDS was the highest contributor to deaths in both groups. Deaths due to pulmonary haemorrhage were significantly more common in babies who died in $2009 / 2010$ compared to those who died in 2000/2001 $(\mathrm{P}=0.002)$. All 7 babies who died due to pulmonary haemorrhage were VLBW babies with underlying RDS, 3 of them receiving surfactant and one having PDA.

Cause specific mortality is shown in Table 8. 
Table 8: Cause specific mortality

\begin{tabular}{|lccccc|}
\hline & $\mathbf{2 0 0 9 / 2 0 1 0}$ & $\mathbf{2 0 0 9 / 2 0 1 0}$ & $\mathbf{2 0 0 0} / \mathbf{2 0 0 1}$ & $\mathbf{2 0 0 0} / \mathbf{2 0 0 1}$ & P value \\
& $n=131$ & Deaths & $n=122$ & $\begin{array}{c}\text { Deaths } \\
\text { No. (\%) }\end{array}$ \\
Respiratory distress syndrome & No. (\%) & No. (\%) & No. (\%) & (\%) \\
Hypoxic ischaemic encephalopathy & $95(72)$ & $11(12 \%)$ & $79(65)$ & $14(18 \%)$ & 0.301 \\
Meconium aspiration syndrome & $08(06)$ & $01(13 \%)$ & $09(07)$ & $09(100 \%)$ & 0.000 \\
Congenital pneumonia & $04(03)$ & 0 & $12(10)$ & $03(25 \%)$ & 0.285 \\
Persistent pulmonary hypertension of newborn & $14(11)$ & 0 & $08(07)$ & $01(13 \%)$ & 0.302 \\
Sepsis & $01(04)$ & $04(80 \%)$ & $01(01)$ & $01(100 \%)$ & 0.292 \\
\end{tabular}

HIE caused significantly more deaths in $2000 / 2001$ compared to $2009 / 2010(\mathrm{P}<0.0001)$

Of the 107 survivors, 2 babies died after discharge from the NICU. Both were VLBW babies with less than 32 weeks gestation; one died of milk aspiration within a week of discharge and the other died at 2 months of age due to a severe episode of bronchiolitis. They were not included in the outcome figures in Table 6 as corresponding figures were not available for the $2000 / 2001$.
Eight babies ( 3 with POG $<32$ weeks, including the 2 deaths) did not attend clinic after discharge. Six were followed up for less than 3 months, one for 3 to 6 months and $92(86 \%)$ till 2 years of age. The 92 included $46(92 \%)$ of 50 babies with POG 32 weeks or less and $46(78 \%)$ of 59 babies with POG over 32 weeks.

Morbidity at the end of 2 years follow up is shown in Table 9.

Table 9: Morbidity at 2 years of age

\begin{tabular}{|lcc|}
\hline \multicolumn{1}{|c}{ Morbidity at 2 years of age } & POG 32 weeks or less & POG more than 32 weeks \\
& Number $(\%)$ & $\begin{array}{c}\text { Number }(\%) \\
\text { Total }=46\end{array}$ \\
No morbidity & Total=46 & $43(93.5)$ \\
ROP self-resolved & $24(52.0)$ & $01(02.2)$ \\
ROP needing laser treatment & $06(13.0)$ & $0(0)$ \\
Reduced visual acuity following laser treatment & $06(13.0)$ & $0(0)$ \\
Squint & $03(06.5)$ & $03(06.5)$ \\
Cerebral palsy & $01(02.2)$ & $02(04.3)$ \\
Infantile spasms & $03(06.5)$ & $0(0)$ \\
Isolated speech delay & $02(04.3)$ & $0(0)$ \\
Poor weight gain & $02(04.3)$ & $0(0)$ \\
Hospital admission for respiratory infection & $02(04.3)$ & $03(06.5)$ \\
Gall stones & $07(15.2)$ & $01(02.2)$ \\
\hline
\end{tabular}

Eight babies had 2 co-morbidities and 4 had 3 comorbidities. There was no morbidity at 2 years of age in significantly more babies with a POG over 32 weeks compared to babies with POG 32 weeks or less $(\mathrm{P}<0.0001)$.

\section{Discussion}

A dramatic fall in neonatal mortality occurred in developed countries with the advent of mechanical ventilation and neonatal intensive care ${ }^{10}$. This has been greater for VLBW infants ${ }^{11}$. This is attributed to increased availability of mechanical ventilation, surfactant and $\mathrm{TPN}^{12}$ and the level of intensive care received $^{13}$. Noninvasive methods provide ventilation without insertion of an ETT. CPAP, the commonest noninvasive mode, applies continuous distending pressure to alveoli throughout the respiratory cycle, maintaining a degree of alveolar inflation during expiration and preventing complete collapse, thus following Laplace law, since a partially inflated alveolus is easier to expand than a fully collapsed one $^{14}$. CPAP may also produce a more regular pattern of breathing in preterm infants by reducing thoracic distortion and stabilizing chest wall, splinting airway and diaphragm, decreasing obstructive apnoea and enhancing surfactant release ${ }^{14}$. In our study CPAP, alone or combined with IMV, was used in $86 \%$ ventilated neonates. CPAP can cause abdominal distension and feeding disturbances because of gas flow to the stomach. Nasal prongs or tubes can cause nasal irritation and excoriation. At high pressures, thoracic air leaks can occur and venous return and cardiac output can be impaired ${ }^{15}$. In our study the 
only complications attributable to CPAP were nasal irritation and feed intolerance.

In IMV breaths are delivered at the rate set by the clinician, irrespective of the baby's breathing efforts. Asynchrony results in inefficiency of gas exchange, gas trapping and air leaks, irregularities in arterial blood pressure, cerebral blood flow velocity and $\mathrm{IVH}^{16}$. In SIMV the onset of inspiration of a mechanical breath is timed to the onset of a spontaneous breath if it occurs within a "timing window" ". In our study, CPAP was the sole mode of ventilation in $33 \%$ and IMV the sole mode in $14 \%$. The outcome in babies receiving CPAP only was significantly better than in those receiving IMV only or in those who received IMV followed by CPAP or CPAP followed by IMV $(\mathrm{P}<0.05)$. Of 88 babies needing invasive ventilation, $27 \%$ needed reintubation. Longer duration of ventilation and higher number of re-intubations were significantly associated $(\mathrm{P}<0.05)$.

Muscle relaxants and sedatives improve synchronization. Use of pancuromium in babies of 26-34 weeks gestation showed that adverse sequelae in the low birth weight ventilated baby was reduced by minimizing periods of non-optimal oxygenation and reducing intracranial pressure ${ }^{17}$. In our study muscle relaxants were not used. Opiates and benzodiazepines are used in ventilated neonates but midazolam has been associated with adverse effects in one study ${ }^{18}$. In our study 54 babies were given midazolam infusion, 19 also receiving morphine and one also receiving phenobarbitone.

Both prophylactic and early surfactant therapy reduce mortality and pulmonary complications in ventilated infants with RDS compared to later selective surfactant administration ${ }^{19}$. A lower threshold $(\mathrm{FIO} 2<0.45)$ to administer surfactant minimizes air leaks and BPD as well as PDA $^{19}$. In our study surfactant was used as rescue therapy in 69 babies, RDS being the indication in $96 \%$. Therapy was initiated when the baby became distressed while on head box oxygen or on CPAP or had increasing oxygen requirement $(\mathrm{FiO} 2>0.30)$ while on nasal CPAP. Significantly more babies with POG 32 weeks or less received surfactant compared to babies with POG over 32 weeks $(\mathrm{P}<0.01)$.

Of the 107 survivors, $92(86 \%)$ babies, were followed up until 2 years of age. There was no morbidity at 2 years of age in significantly more babies with POG over 32 weeks compared to babies with POG of 32 weeks or less $(\mathrm{P}<0.0001)$.
Let us now compare ventilated babies in 2009/2010 and 2000/2001. There were significantly more babies with POG of 32 weeks or less in 2009/2010 ( $<<0.05)$. VLBW babies were significantly more in 2009/2010 $(\mathrm{P}<0.05)$. RDS was the commonest indication for ventilation in both groups. MAS was seen in significantly more babies in $2000 / 2001$ compared to $2009 / 2010 \quad(P<0.05)$. There were no statistically significant differences between the 2 groups regarding duration of ventilation. In 2000/2001, all babies were ventilated invasively whilst in $2009 / 2010$ $33 \%$ were ventilated non-invasively. VAP, nosocomial infection and pneumothorax were significantly more in 2000/2001 $(\mathrm{P}<0.05)$ but ROP was significantly higher in $2009 / 2010(\mathrm{P}<0.01)$. The greater percentage of VLBW babies and their increased survival in 2009/2010 probably accounts for the increased incidence of ROP.

The overall mortality in 2009/2010 was significantly less compared to $2000 / 2001(\mathrm{P}<0.0001)$. Mortality in babies with a POG of 32 weeks or less and mortality in VLBW in 2009/2010 were also significantly less than the corresponding mortalities in 2000/2001 $(\mathrm{P}<0.0001)$. RDS was the highest contributor to deaths in both groups. HIE caused significantly more deaths in $2000 / 2001 \quad(\mathrm{P}<0.0001)$ but pulmonary haemorrhage caused significantly more deaths in $2009 / 2010 \quad(\mathrm{P}<0.01)$. Incidence of pulmonary haemorrhage in VLBW babies is $2-12 \%{ }^{20}$ and the mortality $50-80 \%{ }^{21}$. Risk factors for pulmonary haemorrhage include male sex, prematurity, VLBW, RDS, surfactant use and $\mathrm{PDA}^{22}$. Risk factors contributing to the 6 deaths in our study included VLBW (6/6), RDS (6/6) surfactant use (3/6) and PDA (1/6). Mortality of babies transferred ex-utero in $2009 / 2010$ was significantly more compared to babies transferred ex-utero in 2000/2001 $(\mathrm{P}<0.05)$. This may be due to the higher percentage of babies of 32 weeks gestation or less in 2009/2010 compared to 2000/2001.

In the 2004 study $^{3}$ at General Hospital Matara, common indications for ventilation were: RDS (48\%), MAS (33\%), asphyxia (21\%) and sepsis $(15 \%)$ and the overall mortality rate 52\%. In the 2006 study $^{4}$ at Teaching Hospital Peradeniya, common indications for ventilation were: $\operatorname{RDS}(44 \%)$, birth asphyxia (13\%), MAS (5\%) and sepsis (5\%). Overall mortality rate was $42 \%$ and the mortality rate for RDS was $34 \%$. In our study common indications for ventilation were: RDS (71\%), congenital pneumonia (11\%), asphyxia (6\%), PPHN (4\%), MAS (3\%) and sepsis $(1 \%)$. Overall mortality rate was $20 \%$ and mortality rate for RDS $12 \%$. 
Availability of surfactant, CPAP, parenteral nutrition and improved infection control policies probably contributed greatly to the lesser mortality in 2009/2010.

\section{Conclusions}

- Babies with 32 weeks or less gestation and VLBW babies were significantly more common in $2009 / 2010$ compared to $2001 / 2002(\mathrm{P}<0.05)$.

- Complications such as VAP, nosocomial sepsis and pneumothorax were significantly more common in babies ventilated in 2000/2001 but ROP was significantly more common in babies ventilated in 2009/ $2010(\mathrm{P}<0.05)$.

- HIE caused significantly more deaths in 2000/ 2001 whilst significantly more deaths occurred in ex-utero babies and babies with pulmonary haemorrhage in $2009 / 2010(\mathrm{P}<0.05)$.

- Overall mortality, mortality in babies with a 32 weeks or less gestation and mortality in VLBW babies were significantly lower in 2009/2010 $(\mathrm{P}<0.0001)$.

- In 2009/2010, the outcome in babies receiving CPAP only was significantly better than those receiving IMV only and there was no morbidity at 2 years of age in significantly more babies over 32 weeks gestation compared to babies with gestation 32 weeks or less $(\mathrm{P}<0.0001)$.

\section{Acknowledgements}

We thank Dr. Nalika Gunawardena of the department of Community of Medicine, Faculty of Medicine, Colombo for the guidance provided with regard to analysis. We also thank Dr Lanerolle, Director SJGH, Dr. Rukmal Gunatilataka, Paediatric Registrar SJGH, Nursing Sister and staff of NICU, Nursing Sister of the operating theatre, Mr. Gunawardena, Officer in Charge of Medical Records and his staff and the parents of the babies included in the study for their generous support.

\section{References}

1. Nangia S, Saili A, Dutta AK, Gaur V, Singh M, Seth A et al. Neonatal mechanical ventilation experience at a level II care centre. Indian Journal of Pediatrics 1998; 65: 291-6. http://dx.doi.org/10.1007/BF02752306
2. Trotman H, Barton M, Mitchell V. Outcome of neonates ventilated in the main intensive care unit at the University Hospital of the West Indies: a 15 year experience. Tropical Doctor 2007; 37: 249-50.

http://dx.doi.org/10.1258/004947507782332964

3. Seneviratne TRS, Hewawitharana GP, de Silva MHAD, Wijesekere HKM, Alahakoon CP, Bulegodaarachchi HM. Outcome of neonatal mechanical ventilation in a newly established neonatal intensive care unit. Proceedings of the $12^{\text {th }}$ Asia Pacific Congress of Paediatrics and $2^{\text {nd }}$ Asia Pacific Congress of Paediatric Nursing 2007; 1(1): 41.

4. Bandara S, Jayawardana HMAP. Pattern of diseases among neonates who received IPPV in a tertiary care unit. Programme and Abstracts of the 6th Annual Scientific Sessions of the Perinatal Society of Sri Lanka. June 2007;p 15.

5. Greenough A, Milner AD. Acute respiratory disease. In Rennie JM editor, Roberton's Textbook of Neonatology, 4th ed. Churchill Livingstone;. p. $468-508$.

6. Leuthner SR, Das UG. Low Apgar scores and the definition of birth asphyxia. Paediatric Clinics of North America 2004; 51: 737-45. http://dx.doi.org/10.1016/j.pcl.2004.01.016

7. Gelfand SL, Fanaroff JM, Walsh MC. Meconium stained fluid: approach to the mother and baby. Paediatric Clinics of North America 2004; 51: 655-67. http://dx.doi.org/10.1016/j.pcl.2004.01.012

8. Foglia E, Meier MD, Elward A. Ventilatorassociated pneumonia in neonatal and paediatric intensive care unit patients. Clinical Microbiology Reviews 2007; 20 (3): 409-25. http://dx.doi.org/10.1128/CMR.00041-06

9. Davis JM. Bronchopulmonary dysplasia. In: Sinha SK, Donn SM, editors. Manual of neonatal respiratory care. Armonk, NY: Futura Publishing Co; 2000:310-5.

10. Richardson DK, Gray JE, Gortmaker SL, Goldmann DA, Parsley DM, McCormick MC. Declining severity adjusted mortality: evidence of improving neonatal intensive care. Pediatrics 1998; 102: 893-9. http://dx.doi.org/10.1542/peds.102.4.893 
11. Kaiser JR, Simpson PM, Salhab WA, Rosenfeld CR. Hospital survival of very low birth neonates from 1977 to 2000. Journal of Perinatology 2004; 24:343-50.

http://dx.doi.org/10.1038/sj.jp.7211113

12. St John EB, Carlo WA. Respiratory distress syndrome in VLBW infants: changes in management and outcomes observed by the NICHD Neonatal Research Network. Seminars in Perinatology 2003; 27: 288-92. http://dx.doi.org/10.1016/S0146-0005(03)00056$\underline{9}$

13. Cifuentes J, Bronstein J, Phibbs CS, Phibbs RH, Schmitt SK, Waldemar CA. Mortality in low birth weight infants according to level of neonatal care at hospital of birth. Pediatrics 2002; 109:745-51.

http://dx.doi.org/10.1542/peds.109.5.745

14. Polin RA, Sahni R. Newer experience with CPAP. Seminars in Neonatology 2002; 7(5): 379-89.

http://dx.doi.org/10.1053/siny.2002.0132

15. Attar MA, Donn SM. Mechanisms of ventilatorinduced lung injury in premature infants. Seminars in Neonatology 2002; 7(5):353-60. http://dx.doi.org/10.1053/siny.2002.0129

16. Donn SM, Nicks JJ, Becker MA. Flowsynchronized ventilation of preterm infants with respiratory distress syndrome. Journal of Perinatology 1994; 14(2): 90-4.
17. Finer NN, Tomney RN. Controlled evaluation of muscle relaxation in the ventilated neonate Pediatrics 1981; 67(5):641-6.

18. Hall R, Boyle E, Young T. Do Ventilated Neonates Require Pain Management? Seminars in Perinatology 2007; 31(5): 289-97. http://dx.doi.org/10.1053/j.semperi.2007.07.002

19. Rojas MA, Lozano JM, Rojas MX, Laughon M, Bose CL, Rondon MA, et al. Very early surfactant without mandatory ventilation in premature infants treated with early continuous positive airway pressure: a randomized, controlled trial. Pediatrics 2009; 123(1):137-42 http://dx.doi.org/10.1542/peds.2007-3501

20. Tomaszewska M, Stork E, Minich NM, Friedman H, Berlin S, Hack M. Pulmonary hemorrhage: clinical course and outcomes among very low-birth-weight infants. Archjves of Pediatric and Adolescent Medicine 1999; 153(7): 715-21

21. AlKharfy TM. High-frequency ventilation in the management of very-low-birth-weight infants with pulmonary hemorrhage. American Journal of Perinatology 2004; 21(1):19-26 http://dx.doi.org/10.1055/s-2004-820505

22. Hansen T, Corbet A. Pulmonary physiology of the newborn. In: Taeusch HW, Ballard RA, editors. Avery's diseases of the newborn, 7th ed. Philadelphia, PA: WB Saunders; 1998, pp. 56275. 